RESULTS: Study treatment groups were similar at baseline: SEP-363856 ( $\mathrm{N}=120$; male, 64.2\%; mean age, 30.0 years; PANSS total score, 101.4) and placebo $(\mathrm{N}=125$; male, $63.2 \%$; mean age, 30.6 years; PANSS total score, 99.7). Least-squares (LS) mean reduction from baseline to week 4 was significantly greater for SEP-363856 vs. placebo on the PANSS total score (-17.2 vs. $-9.7 ; \mathrm{P}=0.001$; effect size, 0.45 ), PANSS positive subscale score ( -5.5 vs. $-3.9 ; \mathrm{P}=0.019$; effect size, 0.32), PANSS negative subscale score (-3.1 vs. -1.6; $\mathrm{P}=0.008$; effect size, 0.37), PANSS general psychopathology subscale score ( -9.0 vs. $-4.7 ; \mathrm{P}<0.001$; effect size, 0.51 ), and the CGI-Severity score (-1.0 vs. $-0.5 ; \mathrm{P}<0.001$; effect size, 0.52). Discontinuation rates for SEP-363856 vs. placebo were similar overall (21.7\% vs. $20.8 \%)$ and due to an adverse event (8.3\% vs. $6.4 \%$ ). Change in weight, lipids, glucose and prolactin was similar in SEP-363856 and placebo groups. Adverse events occurring with an incidence $\geq 2 \%$ on SEP363856 or placebo (with SEP-363856 incidence higher than placebo) were: somnolence $(6.7 \%$ vs. $4.8 \%)$, agitation $(5.0 \%$ vs. $4.8 \%$ ), nausea ( $5.0 \%$ vs. $3.2 \%$ ), diarrhea ( $2.5 \%$ vs. $0.8 \%$ ), and dyspepsia ( $2.5 \%$ vs. $0 \%)$. The proportion of patients who reported any extrapyramidal symptom was $3.3 \%$ on SEP363856 and $3.2 \%$ on placebo.

CONCLUSION: In this placebo-controlled study, treatment with SEP-363856, a novel psychotropic agent, was associated with statistically significant and clinically meaningful improvement in schizophrenia symptoms as demonstrated by endpoint change in PANSS total and subscale scores, and CGI-Severity scores. Safety and tolerability findings for SEP-363856 were in general similar to placebo. In particular, SEP-363856 was not associated with extrapyramidal symptoms, akathisia, or hyperprolactinemia, consistent with its non-D2 mechanism of action.

\section{CLINICALTRIALS.GOV: NCT02969382}

Funding Acknowledgements: Supported by funding from Sunovion Pharmaceuticals Inc.

\section{9}

\section{Early Response with Valbenazine and Long-Term Symptom Reduction in Patients with Tardive Dyskinesia: Post Hoc Analysis of the KINECT 3 Study}

Stanley N. Caroff, $M D^{\prime}$; Jean-Pierre Lindenmayer, $\mathrm{MD}^{2}$; Stephen R. Marder, MD ${ }^{3}$; Stewart A. Factor, DO ${ }^{4}$; Khodayar Farahmand, PharmD ${ }^{5}$; and Leslie Lundt, $M D^{6}$

${ }^{1}$ Professor, Department of Psychiatry, Corporal Michael J. Crescenz Veterns Affairs Medical Center and the Perelman School of Medicine, University of Pennsylvania, Philadelphia, PA

${ }^{2}$ Clinical Professor, Department of Psychiatry, New York University School of Medicine, New York, NY
${ }^{3}$ Professor, Department of Psychiatry and Biobehavioral Sciences, University of California Los Angeles, David Geffen School of Medicine, Los Angeles, CA

${ }^{4}$ Professor, Department of Neurology, Emory University School of Medicine, Atlanta, GA

${ }^{5}$ Director, Head of Medical Communications, Neurocrine Biosciences, Inc., San Diego, CA

${ }^{6}$ Medical Director, Medical Affairs, Neurocrine

Biosciences, Inc., San Diego, CA

ABSTRACT: Study Objective: Tardive dyskinesia (TD) is a persistent and potentially disabling movement disorder associated with prolonged exposure to antipsychotics and other dopamine receptor blocking agents. Valbenazine is a highly selective vesicular monoamine transporter 2 (VMAT2) inhibitor approved for the treatment of TD in adults. Using data from a long-term study (KINECT 3; NCT02274558), the effects of once-daily valbenazine (40 mg, $80 \mathrm{mg}$ ) on TD were assessed using the Abnormal Involuntary Movement Scale (AIMS) in participants who were early responders based on subjective measures, including patient self-report (Patient Global Impression of Change [PGIC]) or clinician judgment (Clinical Impression of Change-Tardive Dyskinesia [CGI-TD]).

METHODS: Data from KINECT 3 (6-week double-blind, placebo-controlled [DBPC] period; 42-week double-blind extension) were analyzed post hoc. Long-term outcomes included mean change from baseline to Week 48 in AIMS total score (sum of items 1-7) and AIMS response ( $\geq 50 \%$ total score improvement from baseline) at Week 48 . These AIMS outcomes were assessed in participants who achieved early improvement, defined as a PGIC or CGI-TD score of $\leq 3$ ("minimally improved" or better) at Week 2 (first post-baseline visit of the DBPC period). Participants who initially received placebo were not included in the analyses.

RESULTS: In participants who received only valbenazine (40 or $80 \mathrm{mg}$ ) during KINECT 3 and had available Week 2 assessment, $50 \%(72 / 143)$ had early PGIC improvement (score $\leq 3)$ and $43 \%(61 / 142)$ had early CGI-TD improvement (score $\leq 3$ ). Baseline characteristics were generally similar between participants who achieved early PGIC or CGI-TD improvement and those who did not. Based on available assessments at Week 48, mean AIMS total score change from baseline in participants with early PGIC improvement was similar to those who did not reach the early PGIC improvement threshold (-4.1 $[n=35]$ vs $-3.5[n=41])$. Mean AIMS total score change from baseline in participants with early CGI-TD improvement was similar to those who did not achieve early CGITD improvement $(-4.2[\mathrm{n}=31]$ vs $-3.5[\mathrm{n}=45])$. AIMS response at Week 48 was also similar in those who achieved early PGIC and CGI-TD improvement 
( $40 \%$ and $42 \%$, respectively) compared to those who did not achieve early PGIC and CGI-TD improvement (39\% and $38 \%$, respectively).

CONCLUSIONS: Results from this long-term valbenazine trial indicate that many participants achieved at least minimal patient- and clinician-reported improvement at Week 2. AIMS outcomes at Week 48 demonstrated longterm reductions in TD severity regardless of early response. More research is needed to understand the association between early improvement and long-term treatment effects, but early non-improvement based on subjective measures may not be predictive of long-term treatment failure.

PRESENTED: International Congress of Parkinson's Disease and Movement Disorders; September 22-26, 2019; Nice, France.

Funding Acknowledgements: This study was sponsored by Neurocrine Biosciences, Inc.

140

Effects of Long-Term Valbenazine on Tardive Dyskinesia in KINECT 4: Post Hoc Response and Shift Analyses

Stephen R. Marder, $M D^{\prime}$; Cynthia L. Comella, $M D^{2}$; Carlos Singer, $M D^{3}$;

Khodayar Farahmand, PharmD ${ }^{4}$; and Roland Jimenez, $M S^{5}$

${ }^{1}$ Professor, Department of Psychiatry and Biobehavioral Sciences, University of California Los Angeles, David Geffen School of Medicine, Los Angeles, CA

${ }^{2}$ Professor, Department of Neurological Sciences, Rush University Medical Center, Chicago, IL

${ }^{3}$ Professor, Department of Neurology, University of Miami Miller School of Medicine, Miami, FL

${ }^{4}$ Director, Head of Medical Communications, Neurocrine Biosciences, Inc., San Diego, CA

${ }^{5}$ Sr. Director, Clinical Development, Neurocrine Biosciences, Inc., San Diego, CA

ABSTRACT: Study Objective: Valbenazine (VBZ) is a highly selective vesicular monoamine transporter 2 (VMAT2) inhibitor approved for the treatment of tardive dyskinesia (TD), a persistent and potentially disabling movement disorder associated with prolonged antipsychotic exposure. Post hoc response and shift analyses were conducted using Abnormal Involuntary Movement Scale (AIMS) data from KINECT 4 (NCT02405091), a long-term open-label study in which participants received up to 48 weeks of open-label treatment with once-daily VBZ (40 or $80 \mathrm{mg}$ ).

METHODS: KINECT 4 included participants who met the following criteria: ages 18 to 85 years; DSM-IV diagnosis of schizophrenia, schizoaffective disorder, or mood disorder; neuroleptic-induced TD for $\geq 3$ months prior to screening; stable psychiatric status (Brief Psychiatric Rating Scale score $<50$ ); no high risk of active suicidal ideation or behavior. Stable doses of concomitant medications to treat psychiatric and medical disorders were allowed. VBZ dosing was initiated at $40 \mathrm{mg}$, with escalation to $80 \mathrm{mg}$ at Week 4 based on clinical assessment of TD and tolerability; a dose reduction to $40 \mathrm{mg}$ was allowed if $80 \mathrm{mg}$ was not tolerated. AIMS responses, ranging from $\geq 10 \%$ to $100 \%$ improvement from baseline in AIMS total score (sum of items 1-7), were analyzed at Week 48 based on scoring by site investigators. AIMS shift, conducted for each item (representing 7 different body regions), was defined as an improvement from a score $\geq 3$ (moderate/severe) at baseline to a score $\leq 2$ (none/minimal/mild) at Week 48 .

RESULTS: 103 participants had an available AIMS assessment at Week $48(40 \mathrm{mg}, \mathrm{n}=20 ; 80 \mathrm{mg}, \mathrm{n}=83$ [including 9 with a dose reduction]). At Week $48,94.2 \%$ of participants had $\geq 30 \%$ total AIMS score improvement $(40 \mathrm{mg}$, $90.0 \% ; 80 \mathrm{mg}, 95.2 \%)$ and $86.4 \%$ had $\geq 50 \%$ improvement $(40 \mathrm{mg}, 90.0 \% ; 80 \mathrm{mg}, 85.5 \%)$. The percentage of participants meeting the remaining AIMS response thresholds ranged from $9.7 \%$ (for $100 \%$ response) to $97.1 \%$ (for $\geq 10 \%$ response). In participants who had an AIMS item score $\geq 3$ at baseline, shifts to a score $\leq 2$ at Week 48 were as follows: $100 \%$ for lips, upper extremities, and lower extremities (VBZ $40 \mathrm{mg}$ and $80 \mathrm{mg}$ ). Shift rates for the remaining regions were as follows $(40 \mathrm{mg}$, $80 \mathrm{mg})$ : face $(100 \%$ [9/9], $96.9 \%$ [31/32]), jaw $(100 \%$ [10/10], 97.6\% [40/41]), tongue (100\% [11/11], 97.9\% [47/48]), trunk (87.5\% [7/8], 88.9\% [16/18]).

CONCLUSIONS: After 48 weeks of treatment with oncedaily VBZ (40 or $80 \mathrm{mg}$ ), $>\mathbf{8 5 \%}$ of KINECT 4 participants had a clinically meaningful AIMS response ( $\geq 30 \%$ total score improvement), a robust AIMS response ( $\geq 50 \%$ total score improvement), or an AIMS shift (from item score $\geq 3$ at baseline to score $\leq 2$ at Week 48 ). These results suggest that VBZ is an appropriate long-term treatment for many adults with TD.

Funding Acknowledgements: This study was sponsored by Neurocrine Biosciences, Inc.

\section{1}

\section{Moderating Perspectives of Long Acting Injectable Use of Antipsychotics: A Literature Review}

\section{Kathy Riedford}

Associate Professor, Director of Psychiatric Mental Health Nurse Practitioner Specialty

ANE Primary Care/Mental Health Integrated Model

Project Director

College of Nursing and Health Professions, University of Southern Indiana, Evansville, IN 